\title{
Perspective
}

PERSPECTIVE Actualité en histoire de l'art

\section{2 | 2018}

Détruire

\section{Histoire de l'art et potlatch : regards croisés entre la France et le Canada}

Un débat virtuel entre Sonny Assu, Rémi Labrusse, Marie Mauzé et Charlotte Townsend-Gault, animé par Jean-Philippe Uzel

Sonny Assu, Charlotte Townsend-Gault, Rémi Labrusse, Marie Mauzé et Jean-Philippe Uzel

Traducteur : Françoise Jaouën

\section{(2) OpenEdition}

Journals

Édition électronique

URL : http://journals.openedition.org/perspective/11061

DOI : 10.4000/perspective. 11061

ISSN : 2269-7721

Éditeur

Institut national d'histoire de l'art

Édition imprimée

Date de publication : 31 décembre 2018

Pagination : $37-56$

ISSN : 1777-7852

\section{Référence électronique}

Sonny Assu, Charlotte Townsend-Gault, Rémi Labrusse, Marie Mauzé et Jean-Philippe Uzel, « Histoire de l'art et potlatch : regards croisés entre la France et le Canada », Perspective [En ligne], 2 | 2018, mis en ligne le 30 juin 2019, consulté le 01 octobre 2020. URL : http://journals.openedition.org/ perspective/11061 ; DOI : https://doi.org/10.4000/perspective.11061 


\title{
Histoire de l'art et potlatch : regards croisés entre la France et le Canada
}

\author{
Un débat virtuel entre Sonny Assu, Rémi Labrusse, \\ Marie Mauzé et Charlotte Townsend-Gault, \\ animé par Jean-Philippe Uzel
}

Le présent débat vise à interroger les relations entre l'histoire de l'art et le potlatch sous un nouveau jour. Il est en effet convenu, dans le champ de l'histoire de l'art, de voir dans cette institution cérémonielle en usage chez les Premières Nations de la côte nordouest de l'Amérique avant tout une activité d'échanges somptuaires et de destruction ostentatoire de la richesse. C'est Georges Bataille, dans son texte de 1933 " La notion de dépense ", repris et augmenté en 1949 dans La part maudite", qui s'emploie à développer cette conception spectaculaire du potlatch. Il insiste également sur la dimension éminemment critique du potlatch pour l'idéologie utilitariste des sociétés industrielles, qui sont travaillées par des phénomènes de pertes somptuaires, mais qui refusent de se confronter à cette " part maudite " d'elles-mêmes. Cette conception bataillienne du potlatch a été abondamment reprise par les artistes et les théoriciens de l'art moderne et contemporain, parmi lesquels Miró, Yves Klein, Guy Debord ou Thomas Hirschhorn.

Tout en revenant sur cette fascination du monde de l'art occidental pour le potlatch, le présent débat vise aussi à prendre en considération un élément trop souvent oublié de cette histoire : le point de vue des Autochtones. Il est en effet important de rappeler qu'au moment où les anthropologues, les historiens de l'art et les artistes européens faisaient grand cas du potlatch et y voyaient un principe clef de la création moderne, celui-ci faisait l'objet au Canada d'une stricte interdiction - toute infraction étant punie par la confiscation des objets cérémoniels et l'emprisonnement des organisateurs. C'est ce déni et cette dépossession des cultures des Premières Nations par le pouvoir colonial que nous avons voulu aussi rappeler.

Aussi nous a-t-il semblé nécessaire, pour mener un tel débat, de se situer au croisement de l'histoire de l'art et de l'anthropologie, mais également de faire appel à des spécialistes français et canadiens des relations entre art et potlatch. Nous avons par ailleurs jugé essentiel d'inviter Sonny Assu, artiste contemporain Ligwilda'xw (Kwakwaka $\left.\underline{a}^{\prime} w a k w\right)$ qui évoque dans sa pratique la répression coloniale qui frappa d'interdit le potlatch pendant 67 ans (1884-1951), mais qui s'emploie également à souligner sa persistance chez les Premières Nations de la côte nord-ouest de l'Amérique.

[Jean-Philippe Uzel] 


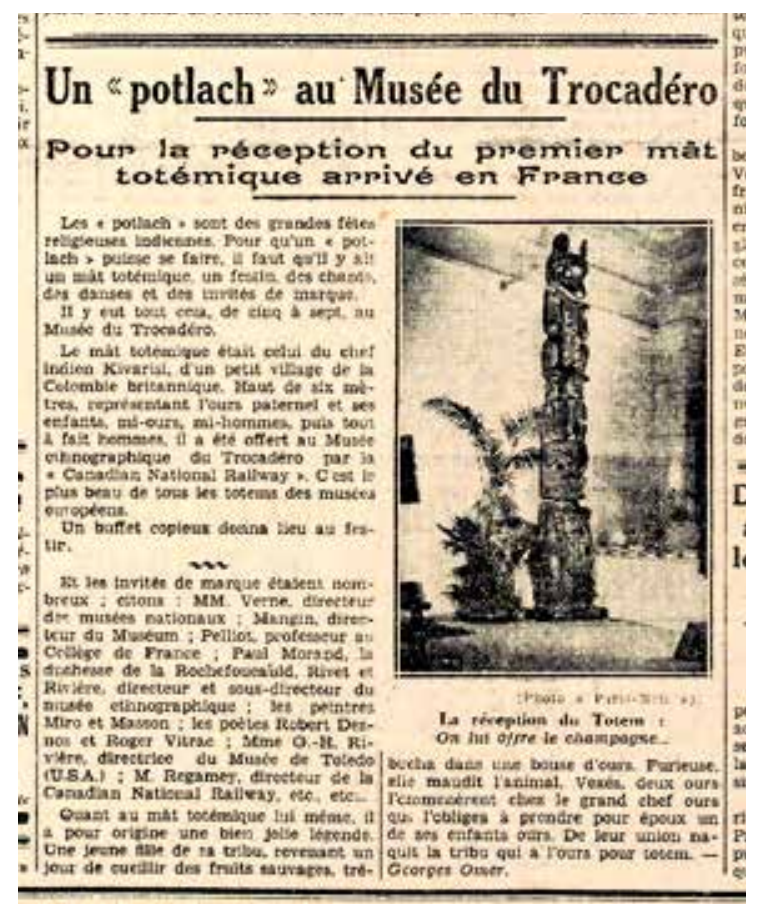

1. Georges Omer, "Un potlatch au Musée du Trocadéro », dans Paris-Midi, 15 mars 1930.
- Jean-Philippe Uzel. On constate que le potlatch est une référence omniprésente dans l'histoire et la théorie occidentales, et tout particulièrement françaises, de l'art moderne et contemporain. Loin de s'essouffler, cette référence semble au contraire se renouveler sans cesse à travers les époques et les courants artistiques : surréalisme, lettrisme et situationnisme, esthétique relationnelle... À quoi attribuez-vous cet attrait des théoriciens et des artistes occidentaux pour le potlatch?

- Rémi Labrusse. Pour caractériser l'irruption de la notion de potlatch dans le champ de l'art occidental pendant l'entre-deux-guerres, il faut commencer par faire droit à ce qu'on peut appeler le tournant anthropologique des avant-gardes à cette période. Le surréalisme n'est pas seul concerné. Parmi les revues européennes, une place à part revient, à partir de 1926, aux Cahiers d'art de Christian Zervos, avec leur ambition de " revue critique et de culture générale » et leur impact international, qui culminent entre 1930 et 1933. Quelles que soient les divergences idéologiques, esthétiques et personnelles entre les milieux, il s'agissait toujours de rendre poreuses les frontières entre créations artistique et intellectuelle

et d'attaquer les fondations de la modernité capitaliste industrielle, en se donnant pour horizon une réinvention anhistorique de nos sociétés, à l'aide de références non-européennes qui concernaient les objets, bien sûr, mais aussi les pratiques sociales et culturelles, les rituels, les modes d'habitation, etc. Nourris de ces références, militantisme refondateur et pensée critique, fétichisme et iconoclasme, révolution et déconstruction se fondaient en un même mouvement À Paris, à la toute fin des années 1930, le Collège de Sociologie de Georges Bataille, Roger Caillois et Michel Leiris a tenté de le dire à travers le concept de sociologie sacrée.

Deuxième série d'éléments explicatifs, cette fois spécifiques à la scène française : la forte tradition américaniste de l'ethnologie française, particulièrement sensible au sein des équipes et donc aussi des collections du musée du Trocadéro, jusqu'à Paul Rivet qui en prend la direction en 1928 ; et, dans ce contexte de moyen terme, l'impact intellectuel de la pensée de Marcel Mauss, grâce à des figures de passeurs du côté des avant-gardes, comme Leiris ou Bataille, mais aussi grâce à l'ouverture d'esprit de Mauss lui-même, qui s'intéressait à l'art contemporain et a intégré à sa réflexion savante un engagement politique et moral affirmé, dénonçant la tyrannie de l'objectivité rationaliste et la transformation de I'homme occidental moderne en " machine à calculer ». Concrètement, les deux milieux - avant-gardes artistiques et sciences humaines - se sont croisés en particulier au musée du Trocadéro : on connaît bien maintenant l'épisode du « potlatch » organisé là-bas en mars 1930, à l'occasion de l'arrivée du mât totémique donné par les Canadian National Railways, cérémonie folklorique très médiatisée (fig. 1) au cours de laquelle Marcel Mauss ou Paul Pelliot (le grand sinologue professeur au Collège de France) ont côtoyé Roger Vitrac, Robert Desnos, Joan Miró ou André Masson, entre autres. 
Cela dit, le contexte n'explique pas tout. "Potlatch », le mot lui-même, le signifiant pur, a certainement joué un rôle important (et il continue de le faire, d'ailleurs) : grande claque sonore qui marque d'autant plus les esprits qu'on ne sait pas très bien ce qu'elle signifie ni d'où elle est partie, qu'elle s'enracine cependant dans un imaginaire primitiviste persistant depuis le XIXe siècle, et qu'elle correspond au goût surréaliste pour les jeux pseudo-magiques du signifiant. Là-dedans se sont agrégées, de façon assez magmatique, une esthétique du don, une esthétique de la destruction et une esthétique de la provocation, selon les procédures de ce qu'Harold Bloom, au début de sa carrière, a appelé la « mésinterprétation créative ${ }^{2}$ » (creative misreading).

- Sonny Assu. Le regard que portent les Occidentaux sur le potlatch est par nature biaisé, puisque la perspective est nécessairement occidentale. Le potlatch est quelque chose de vivant, c'est un vécu. C'est une communauté. C'est donner. C'est le partage de la langue, du chant et de la cérémonie. Il possède également une structure qui peut facilement être négligée par le simple spectateur ou le chercheur et théoricien en histoire de l'art.

Je dirais que ce qui est intéressant, c'est que le potlatch est tout ce que la société, la théorie et les mouvements occidentaux ne sont pas. Le regard occidental est fondamentalement une forme d'appropriation ; il prend sans rien donner en retour et ancre les peuples autochtones, notamment ceux qui pratiquent le potlatch, dans le passé. Comme s'ils avaient depuis longtemps disparu ou qu'ils étaient un simple objet de curiosité négligeable. À cet égard, l'intérêt des Occidentaux manque d'authenticité, et ne correspond pas à la véritable pratique du potlatch.

- Charlotte Townsend-Gault. Ce qui importe, c'est que le potlatch, institution fondamentale et spectaculaire, continue d'exister, et de manière vivace, sur tout le long de la côte, avec des variantes et des échanges (fig. 2a-c). Et c'est cela qu'il faut souligner, afin de compenser son ubiquité référentielle ailleurs, puisque la conception " étrangère » du potlatch persiste également, selon un discours synoptique bâti sur un siècle et demi d'attitudes à l'égard de I'« autre » inconnu, accumulation d'approximations ingénieuses et imaginatives. Elle oscille entre fascination scandalisée de la part de ceux qui ont cru pouvoir mettre un terme à la pratique, et découverte fascinée que d'« autres » façons de faire éclairent ou mettent les nôtres en valeur (qui que «nous » soyons), et ses « objets de désir » complexifient les notions de « valeur» et d'« art».

Au risque de tomber dans la généralisation à outrance, je dirais que le potlatch est de nature collective, relationnelle, coopérative et performative, et la représentation qu'il donne des liens entre humain et surnaturel (terme qui apparaît souvent dans les traductions anglaises des textes issus du travail de Hunt et Boas, tel par exemple que celui cité plus haut) est, d'une manière ou d'une autre, transmissible et transculturelle. Le don, la réciprocité et l'excès - termes associés au discours sur le potlatch - ont connu une seconde vie. Le potlatch était « dialogique » et « relationnel » avant la lettre. Au risque de généraliser une seconde fois, ce ne sont pas les aspects dominants de l'art occidental des deux derniers siècles. Le potlatch est une synthèse entre création et performance. Ses « objets »-masques, tuniques, hochets, bâtons de parole et insignes de pouvoir $-s^{\prime}$ inscrivent tous dans un processus dynamique où ils jouent leur propre histoire. Combinant production et réception, le potlatch renouvelle constamment l'agentivité de ces objets de désir, qui ne sont donc plus des objets de contemplation ni des œuvres d'art isolées. Aucune composante du potlatch n'est « isolée » des autres.

L'histoire des références au potlatch a elle-même produit un riche potlatch, un « agencement collectif d'énonciation», selon la judicieuse formule de Deleuze et Guattari'. Elle est également paradoxale. L'Indian Act de 1884 a interdit le potlatch, considéré comme 

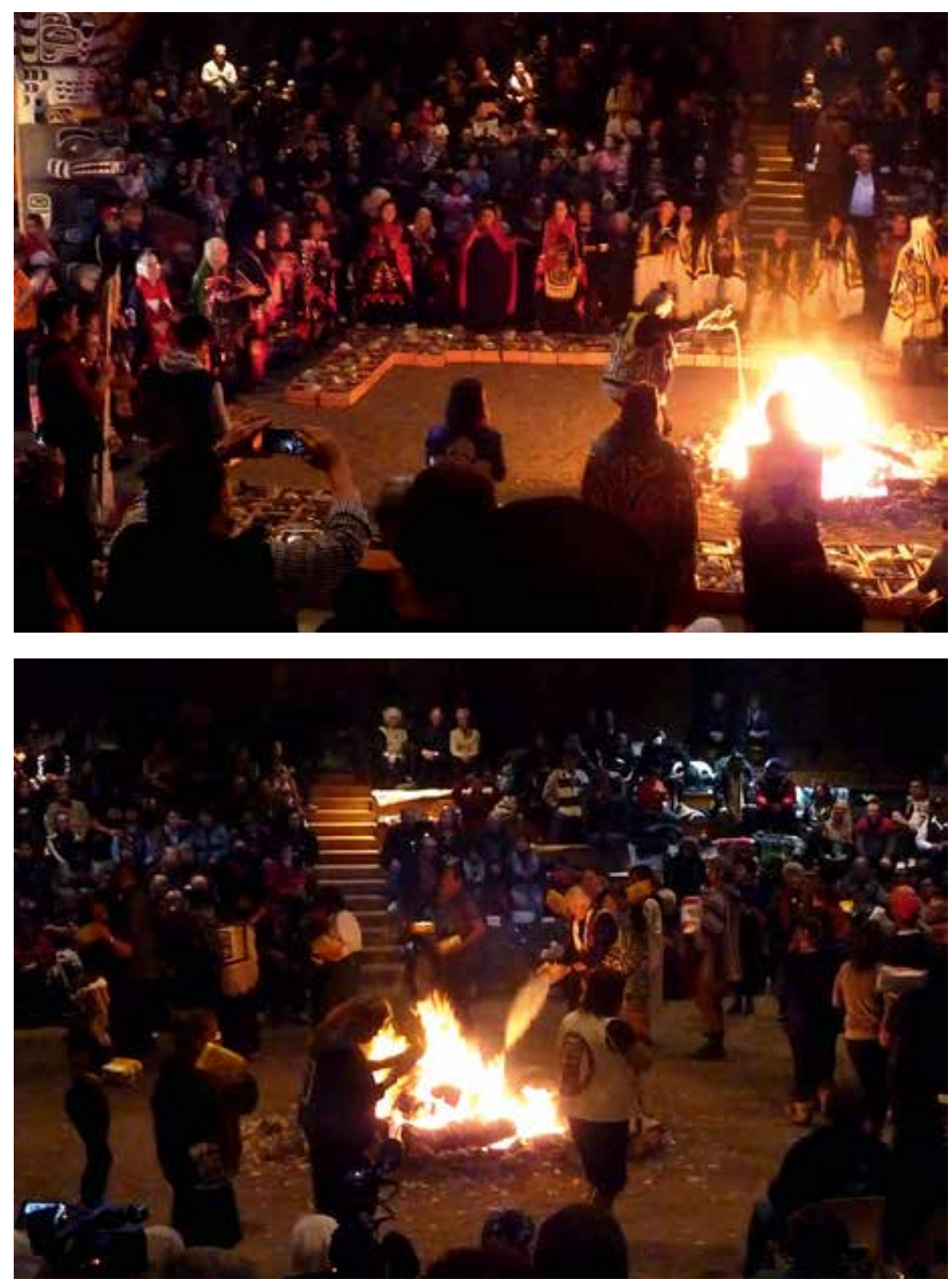

2a-d. Potlatch mémoriel accueilli par Bill Cranmer et Roy Cranmer, les 11 et 12 novembre 2017, à Alert Bay, Colombie britannique (Canada). une menace pour le régime colonial car il débordait I'imaginaire des administrateurs. Les questions qui se posent concernent donc moins les attraits intellectuels et émotionnels du potlatch, qui sont de nature variable, que l'acceptation des opérations de l'imagination humaine. L'histoire de l'art occidental est inconcevable sans I'imagination, même s'il y a toujours eu des tentatives de la contenir, et si le terme n'est plus guère en vogue aujourd'hui. Le potlatch en tant que « référence omniprésente » continue à nourrir l'imaginaire « étranger ». L'exposition Die Macht des Schenkens / The Power of Giving4 qui s'est tenue simultanément au Centre culturel U'mista d'Alert Bay et à la Kunstalle de Dresde en 2011 opérait des rapprochements entre 
les motivations des chefs Kwakwạka ${ }^{\prime}$ wakw et celles des seigneurs de Saxonie à propos du don cérémoniel, montrant des types de dons spectaculaires mais radicalement différents, en s'efforçant de tracer entre eux des parallèles afin de frapper les esprits (fig. 3).

- Jean-Philippe Uzel. Plus le potlatch prend une dimension artistique et esthétique et plus sa signification originelle semble s'effacer. Par exemple, si Georges Bataille dans les années 1930 fait encore quelques références générales à la cérémonie du potlatch chez les Premières Nations, dans la seconde moitié du XXe siècle des auteurs comme Guy Debord ou Nicolas Bourriaud semblent s'en désintéresser. Comment expliquez-vous cet effacement progressif de la nature autochtone du potlatch dans I'histoire de l'art?

- Sonny Assu. La culture occidentale est faite d'effacement et d'exploitation. Si ces auteurs semblent s'en être désintéressés, c'est parce que, ayant pris ce qu'ils cherchaient, ils ont abandonné ce qu'ils pensaient être une coquille vide.

- Charlotte Townsend-Gault. S'effacer pour qui ? De quelle histoire de l'art parle-t-on ? Il est trop tard pour envisager " l'histoire de l'art » sous l'angle binaire autochtone / nonautochtone. Et il est trop facile d'envisager les institutions de l'Autre comme des absolus, ce qui arrive ordinairement lorsqu'on les considère comme intemporelles, et ce qui est aussi le signe d'un imaginaire social défaillant.

On doit se méfier des projets « orientalisants » qui tendent à écarter l'Autre en mettant sa culture au service de fantasmes divers (une appropriation également) - aussi fascinants soient-ils pour certains. Mais la question ne tient pas compte du rôle qu'ont joué les peuples autochtones eux-mêmes pendant des décennies dans le discours sur le potlatch. N'oublions pas que, depuis les années 1880, tout au long d'une période destructrice, ces peuples ont eu des échanges avec les ethnographes et les linguistes, choisissant ce qu'ils voulaient montrer ou aborder, selon leurs propres conditions. Ils ont donc contribué à enregistrer, à préserver et à faire vivre la pratique du potlatch pendant ce que Gloria Cranmer a appelé "les années sombres ${ }^{5}$ ", et au-delà.

On pourrait donc dire qu'ils faisaient une forme d'histoire de l'art. Que l'on songe ici à Doug Cranmer, Ron Hamilton, Art Thompson, Robert Davidson, ou encore Reg Davidson, des artistes reconnus en Occident, et dont l'œurre poursuit, dans des registres différents, ce travail de témoignage, de préservation et de perpétuation. Ils contribuent ainsi à modifier les termes de référence de I'histoire de l'art. À la liste des grands acteurs du « potlatch en histoire de l'art » il faut ajouter Beau Dick, qui s'est fait une réputation internationale avec ses masques, parfois vendus au bénéfice de sa communauté, parfois utilisés dans une danse lors d'un potlatch, ou encore destinés à être brûlés, comme l'ensemble des masques Atlakin. Les archives visuelles de la côte nord-ouest constituent une source pour de jeunes artistes contemporains, qui ne cessent de la modifier.

3. Double visuel de l'exposition The Power of Giving: Gifts in the Kwakwaka ${ }^{\prime}$ wakw Big House from the Canadian Northwest Coast and at the Saxon rulers' court in Dresden, Alert Bay, U'mista Cultural Centre / Dresde, Lipsiusbau, Staatliche Kunstsammlungen Dresden, 2011.

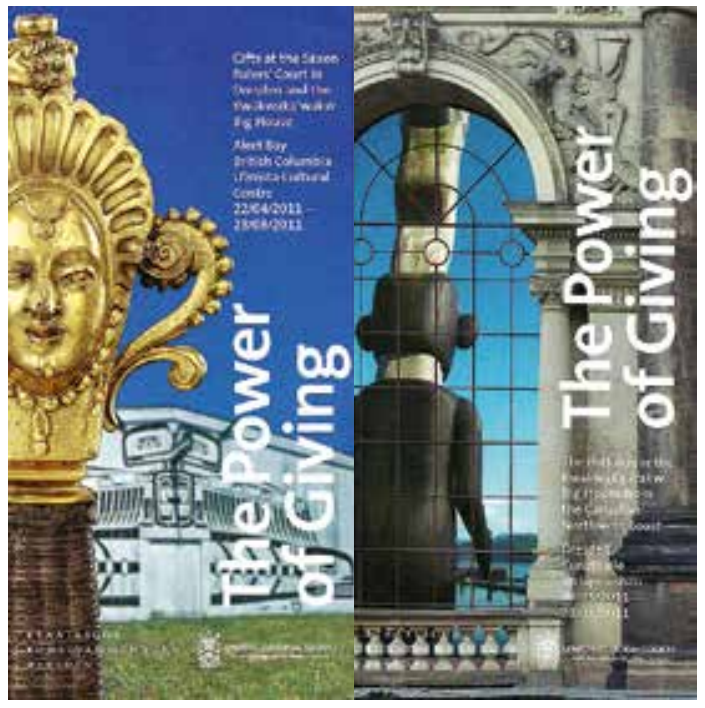


On peut citer ici Marianne Nicolson, Michael Nicol Yahgulanaas, Corey Bulpitt ou Shawn Hunt, qui s'efforcent tous de perpétuer les enseignements visuels de leur culture. Ils puisent ainsi, d'une certaine manière, dans les discours accumulés, dans cet " agencement collectif " des souvenirs et des témoignages des peuples autochtones, et de ceux qui se sont confiés autrefois aux anthropologues et aux historiens et ont été enregistrés. Et, pour autant que je sache, ils s'intéressent aussi, à des degrés divers, aux archives non autochtones.

- Marie Mauzé. Il faut distinguer le « potlatch » en tant qu'institution qui caractérise l'échange de biens matériels et immatériels dans les sociétés de la côte nord-ouest de l'Amérique du Nord, et la notion qui a été popularisée en France à partir des travaux de Marcel Mauss ${ }^{6}$ et à sa suite, ceux de Georges Bataille ${ }^{7}$. Mauss définit le potlatch comme une forme particulière d'échange, à savoir " une prestation sociale de type agonistique ", sous-tendue par un principe de rivalité entre deux collectifs impliqués dans l'échange. Bataille ne retient du potlatch que les aspects extrêmes et les moins couramment attestés, notamment la destruction comme limite de la distribution ostentatoire. Bataille souligne que le potlatch présuppose le mépris de la richesse matérielle qui ne s'en transforme pas moins en désir d'acquisition, de sorte que le potlatch serait une forme de dépense pour un gain de prestige. Au fil d'interprétations successives et dévoyées, le concept " potlatch » est devenu un terme fourre-tout, employé pour faire référence au don somptuaire, au don agonistique, à la destruction, etc. La notion a été aussi utilisée pour critiquer le capitalisme, l'économie de marché, les pratiques de l'art, les formes d'appropriation dans la création. La question se pose de la pertinence de la lecture de Mauss et de Bataille par les historiens et critiques d'art et les artistes. Pour Guy Debord et ses amis, le terme "potlatch », choisi en 1954 pour le titre de la revue de I'Internationale lettriste, se rapporte à la notion de don (somptuaire) : l'envoi gratuit d'un exemplaire du bulletin Potlatch était-il un don " pur » ou valait-il obligation de rendre un contre-don ?

L'emploi du terme "potlatch » a été tellement galvaudé depuis que Mauss s'en est saisi à la suite des travaux de l'anthropologue Franz Boas, qu'il semble vain d'essayer de réconcilier le contexte dans lequel ce terme a été utilisé dans le cadre des activités cérémonielles des sociétés de la côte nord-ouest avec celui dans lequel il est utilisé aujourd'hui par les artistes, les critiques d'art et les historiens de l'art. On est tenté de citer Claude Lévi-Strauss à propos du questionnaire sur L'Art magique que lui avait soumis Breton pour dire que les mots ont une " définition précise » et relèvent " du vocabulaire ethnologique ", qu'il s'agisse de potlatch, de magie, ou de sacré ${ }^{8}$.

- Rémi Labrusse. L'arrachement du potlatch à sa signification originelle amérindienne et son appropriation intégrale à des fins de critique de la société occidentale contemporaine, par les milieux d'avant-garde, est un phénomène tout à fait fascinant. Après Bataille, le cas de Guy Debord (en attendant d'évoquer l'art actuel tout à l'heure) est particulièrement parlant. Comme on sait, après avoir rompu avec les lettristes d'Isidore Isou, il contribue à fonder I'Internationale lettriste en 1953 et à créer la revue Potlatch, qui connaît 29 numéros (en fait 27 livraisons compte tenu d'un numéro triple) entre juin 1954 et novembre 1957. Ce qui est frappant, c'est son écartement délibéré de la source amérindienne du mot. Dans le numéro 14, Potlatch annonce une de ces fausses " enquêtes » qui tournaient en ridicule un genre en vogue dans le surréalisme. En l'occurrence, la question est : «Qui est Potlatch ?» Puis le lecteur a le choix entre trois réponses : " un espion soviétique »; " une pratique du cadeau somptuaire, appelant d'autres cadeaux en retour, qui aurait été le fondement d'une économie de l'Amérique précolombienne »; et " un vocable vide de sens inventé par les lettristes » (fig. 4). Enfin vient, dans le numéro suivant, le faux résultat de cette fausse enquête : "Les opinions les plus répandues sont exprimées par le troisième cas : vocable 
vide de sens (Franc-Tireur, Camus, etc.) et le premier cas : espion soviétique (Aspects de la France, Breton, G. Mollet, etc.). Cependant quelques personnes parmi nos correspondants soutiennent hardiment la deuxième éventualité : cadeau somptuaire. II est donc inutile de s'attarder sur ce problème, aussi embrouillé que tous les problèmes que cette société feint de se poser ${ }^{9}$. " Autrement dit, l'anti-primitivisme de Debord et de ses amis les conduit à effacer ironiquement toute pensée culturaliste au profit d'un détournement de " potlatch » en tant que mot d'ordre révolutionnaire (et non pas $d u$ potlatch en tant que réalité culturelle des Premières Nations).

Dans ce cadre, qu'est-ce qui rapproche l'Internationale lettriste de ses antécédents batailliens ? La fusion, sous le signe de Potlatch, de la déconstruction critique et de l'esthétique de la destruction et, à partir de là, la volonté de travailler « à l'établissement conscient et collectif d'une nouvelle civilisation ${ }^{10} »$. Mais qu'est-ce qui la différencie ? Le rejet de toute généalogie ethnologique, a priori suspectée de sentimentalisme primitiviste, et la construction de la notion de jeu à partir d'une éthique paradoxale de la dérive et du provisoire. La situation, en tant que « forme de vie » ou " manière de vivre [...] qui tend elle-même à ne s'exercer que dans le provisoire ${ }^{11}$ », exclut la possibilité d'un horizon sacré ou cérémoniel, lequel restait central dans les approches surréalistes et batailliennes du potlatch. À la fête somptuaire succède le jeu à la dérive. Consécutivement, Potlatch - la revue - se concentre sur une critique révolutionnaire du capitalisme marchand, alors que, dans l'entre-deux-guerres - dans Documents, Acéphale ou Minotaure -, on s'attaquait plutôt et plus généralement au désenchantement moderne du monde, via le mot d'ordre d'une « sociologie sacrée ». Le potlatch version Debord est déraciné de son sol natal, désethnologisé, désacralisé, désaristocratisé, si l'on peut dire. Cet effacement de la «nature autochtone » du potlatch, comme vous dites, est parfaitement conscient : ce n'est pas seulement un phénomène banal d'appropriation de l'ailleurs, pour faire flamboyer poétiquement l'esthétique de la destruction; il s'agit aussi et surtout d'une position critique anti-primitiviste, qui prend acte de la mondialisation de l'économie du spectacle et qui légitime l'anti-colonialisme intégral de l'Internationale lettriste.

- Jean-Philippe Uzel. La lecture que Georges Bataille fait du potlatch, à partir de l'Essai sur le don de Marcel Mauss, met l'accent sur la destruction des biens et des richesses. Cette lecture « sensationnaliste » a eu un impact immédiat sur les avant-gardes, par exemple chez Miró, mais n'est-elle pas une distorsion de la vision du monde autochtone ? Par exemple, détruire des coppers (écussons de cuivre de grande valeur) au cours d'un potlatch est aussi chez les Premières Nations de la côte nord-ouest de l'Amérique du Nord (Kwakwa $\underline{a} \underline{a}$ 'wakw, Haïda, Tsimshian...) un signe de justice et de réparation. Est-ce que réduire le potlatch à une destruction des biens et des richesses n'est pas finalement une vision réductrice de celui-ci?

- Sonny Assu. Il s'agit là d'une interprétation foncièrement réductrice. Le regard occidental a, une nouvelle fois, ramené un exemple singulier et rare de potlatch à son aspect exclusivement sensationnel pour le rendre conforme à sa vision. La distribution de richesses est une 
dimension essentielle du potlatch, mais la destruction de richesses (des écussons de cuivre) est très rare. C'est un geste d'une grande portée qui nécessite mûre réflexion.

La fascination des Occidentaux pour la destruction de richesses matérielles est probablement liée à la culture de consommation, qui accorde une valeur à des objets inanimés qui n'en ont souvent aucune en eux-mêmes. La valeur est une construction mentale et elle est subjective. Le copper est un témoignage conceptuel qui contient la valeur de ce que le chef a donné au cours du potlatch. C'est un témoignage oral qui doit être énoncé de sorte à augmenter ou diminuer sa valeur à chaque potlatch. Le cuivre est un matériau, et c'est le regard occidental qui considère l'écusson comme une richesse matérielle, alors que sa richesse est en réalité d'ordre conceptuel. C'est pour cela, selon moi, qu'un chef n'hésite pas à le détruire ou à le briser, accomplissant ainsi une forme de justice ou d'attrition.

- Marie Mauzé. C'est à partir de l'analyse de l'institution du potlatch que Bataille élabora sa théorie économique centrée notamment sur la notion de consumation, inhérente pour lui à I'utilisation non-productive des ressources, cette notion s'opposant à celle de l'accumulation. Sa théorie s'appuie sur L'Essai sur le don de Mauss, qui lui-même a fondé sa recherche sur les travaux de l'anthropologue Franz Boas sur les Kwakwákáwwakw (Kwakiutl) de la côte nord-ouest de l'Amérique du Nord ${ }^{12}$. Pour Mauss, le potlatch est un " phénomène social total » concernant l'ensemble de la société et de ses institutions. Le potlatch n'apparaît plus comme un phénomène purement économique. Cependant, Mauss s'attache aux échanges au cours desquels s'expriment les antagonismes entre rivaux, auxquels il donne le nom de "prestations totales de type agonistique ${ }^{13}$ ", et note que dans le potlatch " la consommation et la destruction [...] sont sans bornes ${ }^{14} »$. En privilégiant certains faits ethnographiques auxquels il a donné plus d'importance qu'ils n'en ont dans le contexte global des sociétés de la côte nord-ouest - les destructions de biens sont plus spectaculaires chez les Kwakwákáa'wakw qu'au sein des autres sociétés de la région - Mauss a contribué à fausser la compréhension du potlatch. Il a mis en lumière les aspects les plus spectaculaires associés au développement du potlatch dans le contexte colonial marqué par l'arrêt des guerres intertribales, une dramatique diminution de la population, l'introduction des biens européens, la monétarisation des revenus, autant de phénomènes qui ont altéré la nature même de l'institution.

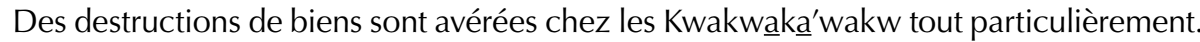
Les cuivres étaient l'expression suprême de la richesse. Ils opéraient comme des biens précieux quand ils étaient conservés au sein des groupes (clans, etc.). Ils fonctionnaient comme substituts d'esclaves tués à la mort de leur maître ou ensevelis à l'occasion de l'érection de mâts funéraires et, à ce titre, dans un rapport d'équivalence en tant que biens de prestige, pouvaient être donnés, détruits, notamment lors des potlatchs funéraires. Dans le cadre des potlatchs de rivalité, les cuivres étaient détruits, ou noyés. Certains cuivres avaient aussi valeur de monnaie.

À travers la filiation Boas-Mauss-Bataille, c'est une vision biaisée et simplifiée du potlatch qui s'est progressivement formée. À cela s'ajoute le fait qu'un seul terme « potlatch », issu du sabir chinook, utilisé par les Blancs, désigne un certain nombre de manifestations distinctes au cours desquelles étaient distribués des biens.

- Rémi Labrusse. Bataille s'est en effet fondé sur les énoncés les moins ethnographiques, les plus philosophiques et, en même temps, les plus militants de l'Essai sur le don, où Mauss reconduit la dimension donatrice, dans le « fait social total » du potlatch, à une dimension destructrice revêtue, à ses yeux, d'une primauté conceptuelle (" donner, c'est déjà détruire »). Ce faisant, le gain en pertinence politico-philosophique, dans la construction moderne du concept de potlatch, se fait au prix d'une perte en pertinence ethnologique. 
Cela dit, je voudrais apporter deux nuances. La première, purement historique, c'est que Bataille ne peut pas faire figure de point de départ pour cette exaltation esthético-morale de la destruction. Depuis le début des années 1920 à Paris, depuis le premier Max Ernst dada et le jeune André Breton lecteur de Marcel Schwob (" détruis, car toute création vient de la destruction $\left.{ }^{15} »\right)$, parmi tant d'autres, l'exaltation de la destruction est un motif obsessionnel des avant-gardes. Masson, grand blessé de la Grande Guerre, effrayait ses amis par ses transes auto-destructrices, dans son atelier de la rue Blomet, dès 1922, et Miró proclamait, en 1927, qu'il voulait « détruire l'industrie picturale ${ }^{16}$ » et " assassiner la peinture $^{17} »$. Dans ce paysage intellectuel, l'apport un peu plus tardif de Bataille est d'avoir fait du potlatch, en tant que tel, un étendard, un mot de ralliement. Pour ce faire, il l'a détaché de son contexte culturel d'origine et a accentué sa valeur anthropologique générale, afin qu'il devienne l'emblème poétique du concept de dépense sacrificielle. Ultimement, chez Bataille, les réflexions anthropologiques - fondées sur Mauss et Huizinga ${ }^{18}$ - ont d'ailleurs elles-mêmes une fonction instrumentale et l'horizon de la pensée, comme on sait, est une contre-métaphysique d'inspiration nietzschéenne.

La seconde remarque, c'est que, dans l'esprit de la nébuleuse bataillienne des années 1930, quelle que soit l'imprécision, voire l'occultation, du contexte des sociétés amérindiennes, les usages du potlatch, en tant que notion poético-philosophique, visent clairement une forme "de justice et de réparation ", selon les termes de votre question, sur le plan social et symbolique. Ils sont articulés à un désir de rénovation de la pensée du sacrifice, avec une dimension à la fois politique et parareligieuse. La justice politico-sociale y est impliquée à travers la dénonciation de l'exploitation capitaliste (Bataille publie « La Notion de dépense » dans La Critique sociale, la revue communiste démocratique de Souvarine). Et l'horizon de la réparation se donne aussi à penser comme un espoir de transmutation de l'objectivisme matérialiste occidental en pure subjectivité tragique. C'est pourquoi, dans les années 1950, Bataille ira jusqu'à considérer les armes de destruction massive comme la seule et ultime forme de sacré par laquelle les sociétés contemporaines font craquer l'économie restreinte du gain matériel. La force de la notion de potlatch, dans ce cadre, est d'avoir permis l'énoncé d'une réparation à la fois politique et « mystique » (c'est un terme que Bataille et ses amis affectionnent) sur des prémisses strictement matérielles : l'enjeu (ou l'utopie), en somme, est celui d'une réconciliation inouïe entre matérialisme philosophique et sacralité archaïque.

- Charlotte Townsend-Gault. Le fait que ces interprétations réductrices connaissent une certaine vogue démontre notamment que l'histoire de l'art et l'anthropologie s'accordent mal, voire n'ont aucun lien. Ces interprétations sont, entre autres, des constructions souples, discrètes et imaginatives qui ne s'embarrassent guère des faits. Et la distorsion est un synonyme - pas nécessairement déshonorant - du jeu de l'avant-garde, pour lequel il est plus important de se saisir d'une idée que d'en avoir une conception juste, ou de se livrer à une analyse (ethnographique ; contraignante et hors de propos). Puisque la pratique du potlatch semble transgresser les règles connues, la transgression fait partie de son attrait.

Le témoignage le plus complet nous vient de l'ethnographe tsimshian William Beynon, qui assista à un potlatch à Gitsegukla pendant la période de l'interdit. Il a fallu attendre I'an 2000 pour que ses carnets soient publiés, sous le titre Potlatch at Gitsegukla: William Beynon's 1945 Field Notebook ${ }^{19}$. Je rappelle que, selon certaines interprétations que I'on peut qualifier de révisionnistes, le potlatch est une pratique controversée au sein même des sociétés autochtones. C'est ce qu'affirme notamment l'ouvrage collectif Standing up with Ga'axsta'las: Jane Constance Cook and the Politics of Memory, Church and Custom ${ }^{20}$, ouvrage dans lequel les descendants de cette figure controversée évoquent l'impact qu'a eu 
le christianisme sur les comportements, notamment celui des femmes, vis-à-vis des hiérarchies de pouvoir dans la communauté des familles pratiquant le potlatch et au sein de celles-ci.

- Jean-Philippe Uzel. Le fait que l'histoire de l'art européenne se soit intéressée au potlatch au moment où celui-ci faisait l'objet d'une interdiction au Canada (de 1884 à 1951), ne nous oblige-t-il pas à réviser en profondeur cette lecture occidentale qui a largement passé sous silence la « destruction » de la culture autochtone par le pouvoir colonial ?

- Charlotte Townsend-Gault. On comprend sans doute mieux aujourd'hui que la fascination " étrangère " pour le potlatch a été exacerbée par le fait que les missionnaires, entre autres, prompts à se méprendre, estimaient qu'il constituait une transgression morale et une entrave à la colonisation. Breton et d'autres surréalistes ont donné à cette fascination un caractère d'extraordinaire, et Bataille une dimension transgressive. Il est devenu banal de réorienter la réflexion sur le mode du " politiquement correct ». Mais la révision est indispensable si I'on veut repenser les relations coloniales, voire les réparer si cela se peut. Les débats sur l'art ou l'amélioration des relations ne doivent pas faire oublier que le racisme reste fortement ancré au Canada comme aux États-Unis. Il s'agit là d'un point essentiel aujourd'hui, puisqu'une forme d'orientalisme est à l'œuvre sur la côte nord-ouest depuis des décennies, voire des siècles, avec tous les aveuglements trompeurs que le terme implique. La pratique du potlatch comporte depuis toujours un aspect spectaculaire qui vise à captiver un public, à impressionner et même interloquer, ou encore divertir. Mais on note aussi que la situation peut parfois s'inverser, et que certains éléments de la " mésinterprétation »sont repris, absorbés et tournés en dérision. Americans, l'exposition qui se tient en ce moment au National Museum of the American Indian à Washington ${ }^{21}$, cherche à montrer que l'Amérique a incorporé de manière permanente les idées, les objets et les références des cultures autochtones qu'elle a écrasées presque au point de les faire disparaitre. Mais elles n'ont pas disparu. Elles sont partout, comme en témoigne cette exposition à la fois sombre et paradoxalement hilarante.

- Rémi Labrusse. Le surgissement du potlatch dans I'horizon de l'art européen moderne s'inscrit dans le cadre plus vaste d'une sensibilité primitiviste où une persistante mélancolie concurrence, voire menace, l'engagement politique concret, y compris dans des cercles radicalement anti-occidentaux comme Le Grand Jeu, à Reims, en 1928-1929, autour de Roger Gilbert-Lecomte, Roger Vailland et René Daumal. Quelque incontestable que soit I'anticolonialisme des avant-gardes à cette époque, il ne conduit pas tant à un engagement structuré, pour sauver de la destruction coloniale des réalités culturelles comme le potlatch, sur leurs terrains d'origine, qu'à un désir d'appropriation et de réinvention de ces mêmes réalités en Europe même. Dont acte, sur un plan politico-moral.

De même, l'occidentalisation systématique des voies d'approche du potlatch (parmi d'autres cas analogues) affaiblit, voire annule, la pertinence ethnologique d'une telle lecture, sur un plan, disons, scientifique. On se plaît parfois, dans l'histoire de l'art contemporain, au jeu des analogies, en rapprochant la mise en avant de l'inappropriable, de l'éphémère, de l'immatériel ou du bassement matériel, dans la création occidentale contemporaine (qu'on pense aux collages et aux assemblages de Miró, au tournant des années 1920 et 1930), et le mépris affiché de la richesse matérielle, dans les potlatchs des Premières Nations ; ou encore la provocation dans l'art contemporain - pensez à l'art de « l'échange d'injures » chez les situationnistes - et les joutes d'insultes dans certaines cérémonies assimilables au potlatch. Mais il n'est pas besoin d'être très savant pour sentir la faiblesse structurelle de telles analogies, qui relèvent clairement d'une forme de néo-primitivisme, bien plus que d'une vérité psycho-sociale. 
Donc, réviser en profondeur la valeur de vérité ethnologique des lectures occidentales du potlatch, dans le champ de l'histoire de l'art en particulier, oui, c'est le moins qu'on puisse faire - mais en gardant toujours en vue l'efficacité interne de cet étrange déracinement, de cette transmutation du potlatch en instrument conceptuel intégralement occidental. Par le son autant que par le sens, il cristallise cette pulsion autocritique de la modernité occidentale, que les avant-gardes ont portée, et qui impliquait par nature un mouvement centripète $d$ 'instrumentalisation de tous les combustibles venus d'ailleurs.

- Marie Mauzé. De fait, comme je l'ai déjà souligné, les historiens de l'art se sont intéressés à un concept et non aux sociétés auxquelles ce concept était associé. Cependant, deux artistes de la constellation surréaliste, Kurt Seligmann et Wolfgang Paalen qui se sont rendus en Colombie Britannique respectivement en 1938 et 1939, ont tous les deux dénoncé les conditions faites par le pouvoir colonial aux cultures autochtones, avec l'interdiction du potlatch, institution centrale de l'organisation sociale et cérémonielle de ces sociétés. Ils avaient également une perception aiguë des conséquences de cette interdiction sur
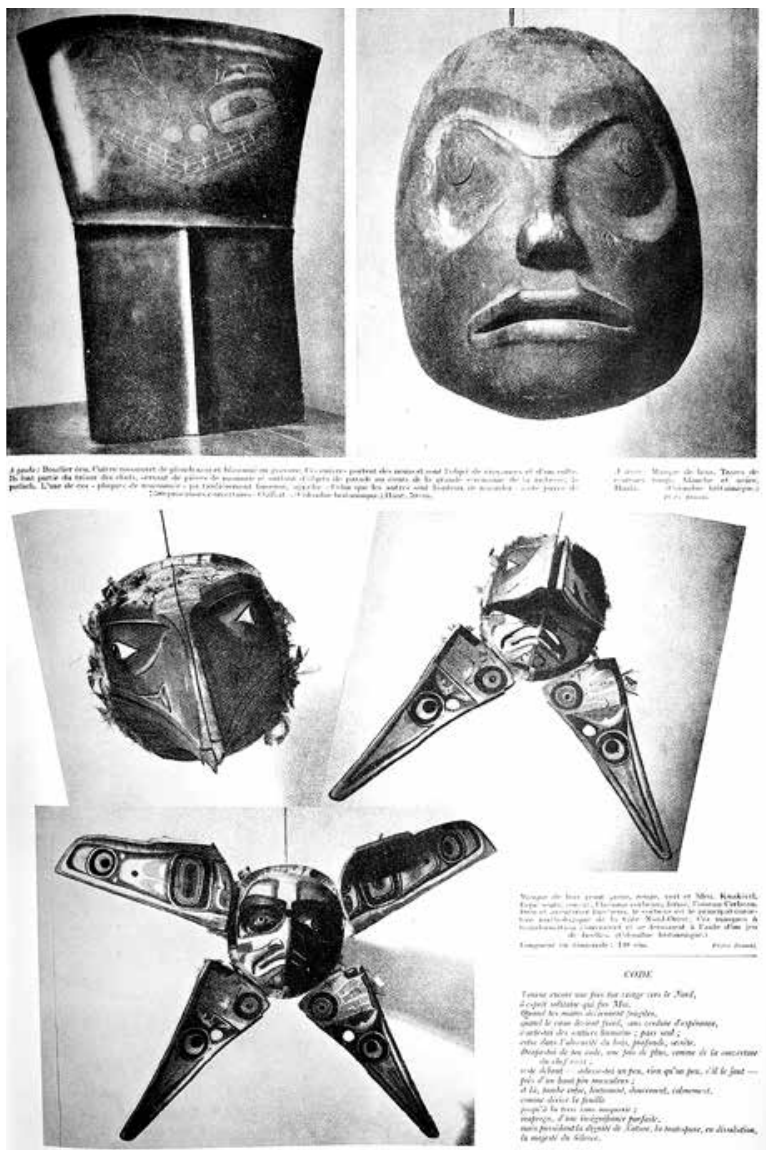

5. Georges Duthuit, "Le don indien ", dans Labyrinthe, $\mathrm{n}^{\circ} 18,1^{\mathrm{er}}$ avril 1946, p. 11. la production d'objets à usage cérémoniel. Quant au critique d'art Georges Duthuit, qui a aussi fait partie de la mouvance surréaliste, notamment pendant la Seconde Guerre mondiale, lors de son exil américain, il a défini dans Le Don Indien ${ }^{22}$ le potlatch comme « une sorte d'opéra cosmique qui tient du culte, du carnaval et du marché, du sacrifice et de la réception mondaine sublimée ${ }^{23}$ ", s'inscrivant ainsi dans les pas de Bataille (fig. 5). À sa manière, Duthuit a condamné aussi les effets délétères de l'administration coloniale et du christianisme qui, au nom de la « décence et de la moralité » ont transformé ces grands hommes en hommes de rien ${ }^{24}$. En se focalisant sur une théorie, un concept, l'histoire de l'art a oblitéré l'histoire des sociétés dont ce concept est originaire, les conflits auxquels les peuples autochtones ont été confrontés dans le passé et les batailles qu'elles livrent aujourd'hui.

- Jean-Philippe Uzel. On constate que les artistes des Premières Nations ont joué un rôle de premier plan pour la conservation et la résurgence du potlatch, du livre de George Clutesi Potlatch ${ }^{25}$ jusqu'aux performances-cérémonies Awalaskenis I et Awalaskenis II (2013-2014) de Beau Dick. Aujourd'hui de très nombreux artistes contemporains autochtones font référence au potlatch dans leur pratique. Comment interprétez-vous ce mouvement de résurgence du potlatch chez les artistes autochtones? 


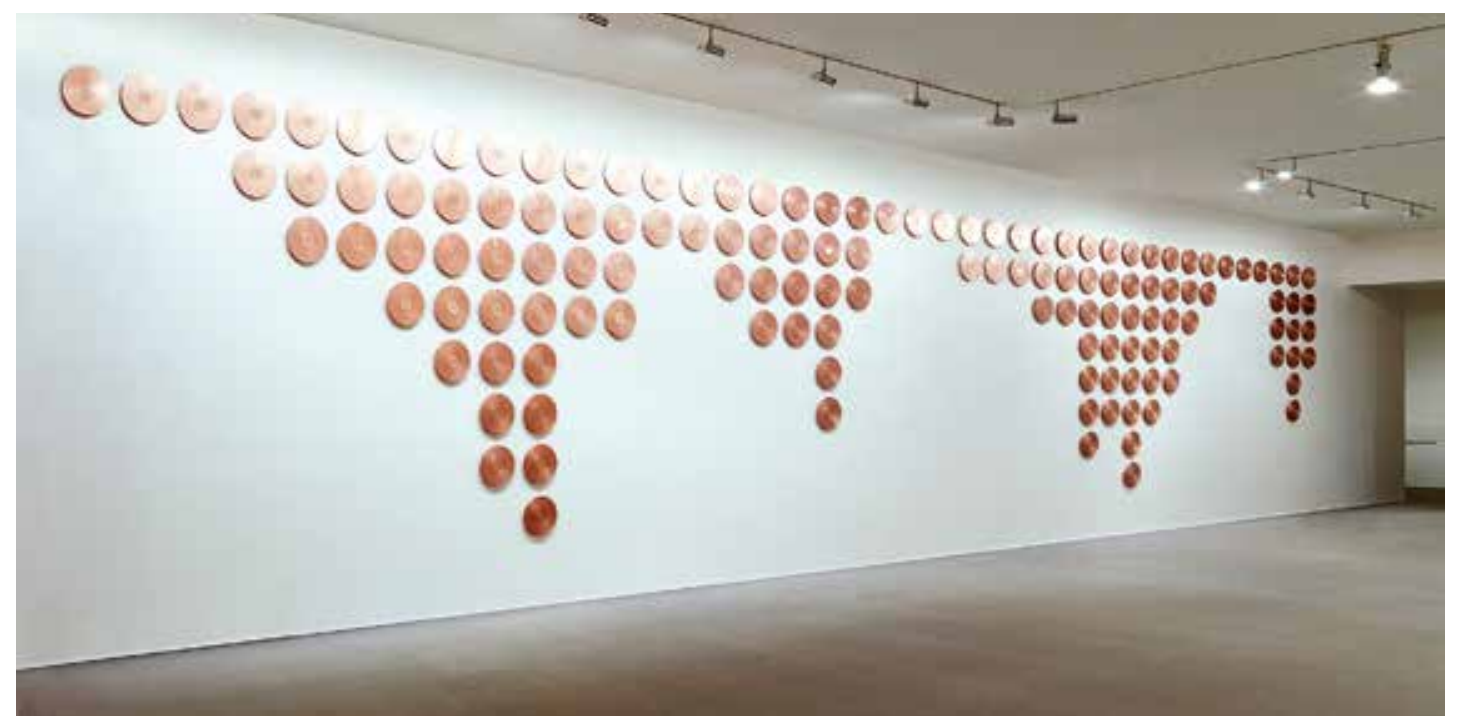

- Sonny Assu. Les artistes autochtones ont joué un rôle clef en écartant le potlatch de la vision occidentale et du paradigme du sauvage. Parler de résurgence semble indiquer que le potlatch a disparu pendant un temps. Or la pratique s'est poursuivie, même pendant la période d'interdiction par le régime colonial. Quant à moi, j'utilise le potlatch de manière conceptuelle. C'est une façon de raconter ce qui est arrivé aux peuples autochtones des territoires qui forment aujourd'hui le Canada, et ce qui est arrivé à ma famille (fig. 6a-b). Les artistes autochtones actifs aujourd'hui, notamment ceux qui se réfèrent, en le détournant, au discours occidental, proposent une vision plus authentique du potlatch. Ces artistes, dont je fais partie, n'éprouvent pas la fascination des surréalistes ou d'autres, parce que leur inter-

6a-b. Sonny Assu, Ellipsis [a], et détail [b], installation de 137 disques de cuivre, 2012, collection de l'artiste.

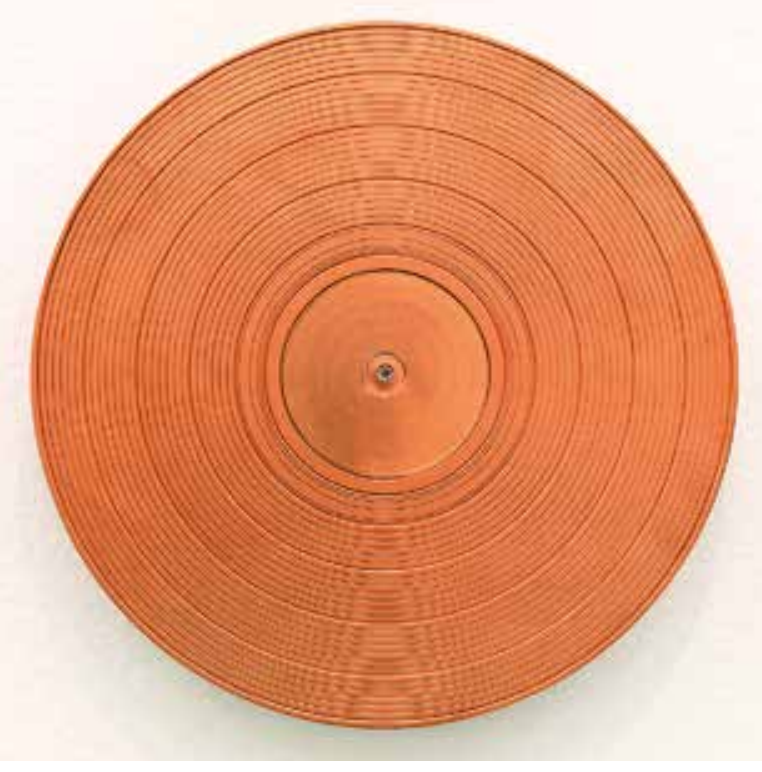
prétation du potlatch est fondamentalement creuse. Ils s'intéressent à l'« autre » dans la mesure où cette résurgence, comme vous l'appelez, perpétue l'authentique.

- Marie Mauzé. Lorsque la Loi sur les Indiens a été modifiée en 1951 et que des potlatchs ont été à nouveau organisés par les chefs titulaires de droits et de privilèges, ces derniers ont fait appel aux artistes locaux pour sculpter, par exemple, des masques et des mâts héraldiques. Aujourd'hui, les sculpteurs produisent des œuvres à la fois pour un usage cérémoniel et pour un usage commercial destiné à des consommateurs non autochtones. Beau Dick, un chef héréditaire kwakwa $k^{a}{ }^{\prime} w a k w$ et un sculpteur de grande renommée, a formé une nouvelle génération d'artistes. Les deux performances qu'il avait organisées en 2013 et 2014 s'inscrivaient dans le mouvement de 


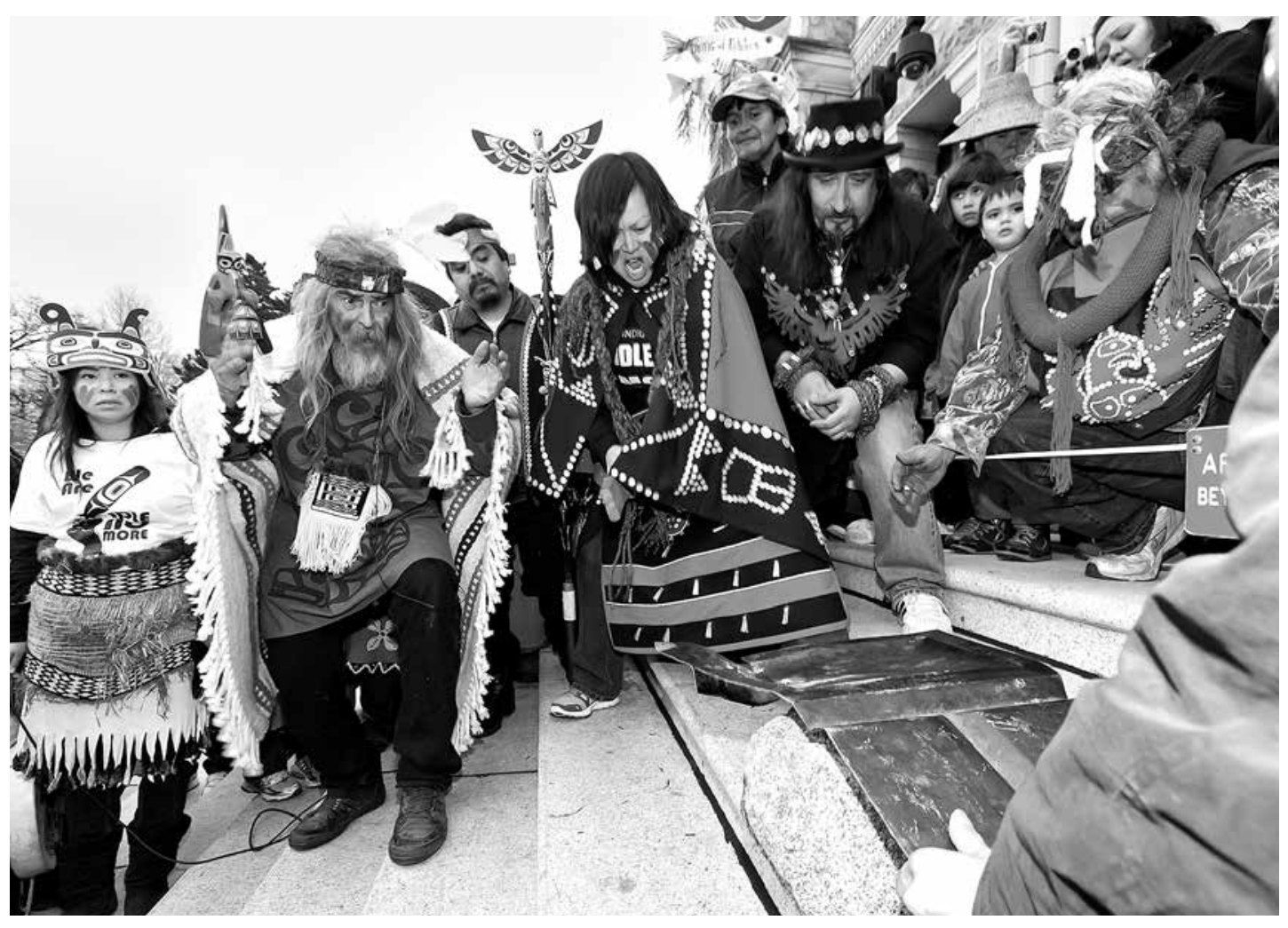

contestation Idle No More mené par les Premières Nations du Canada (Inuit et Métis compris) face aux politiques menées par le gouvernement fédéral avec le projet de 7. Awalaskenis I. Beau Dick et d'autres participants brisant le cuivre sur les marches du Parlement de Colombie loi C-45 (2012) affectant notamment les droits de l'eau britannique, 10 février 2013. et les droits fonciers reconnus par la Loi sur les Indiens (1951). Les Autochtones dans tout le Canada avaient rallié ce mouvement de protestation. Sur la côte ouest, Beau Dick a été l'un des acteurs principaux de ce mouvement et a entrepris, aux côtés de militants autochtones, une action spectaculaire au terme d'une marche de près de 500 km : le bris d'un cuivre kwakwa kaka'wakw sur les marches du Parlement de la Colombie britannique à Victoria en 2013, devant un public très nombreux (fig. 7). Cet acte symbolique a été renouvelé quelques mois plus tard, à la suite d'un voyage qui a mené les protestataires de Vancouver à Ottawa, devant le Parlement canadien, où Beau Dick a brisé un autre cuivre qui lui avait été transmis par le sculpteur et militant haïda, Gujaaw. En s'emparant d'un cuivre, symbole par excellence de richesse et de statut social parmi les peuples autochtones de la côte nord-ouest, Beau Dick puise dans les traditions culturelles les plus anciennes pour défier un gouvernement qui ne respecte pas la souveraineté des Premières Nations et la protection de l'environnement. Ce qui est dans son contexte d'origine une action rituelle opposant deux chefs kwakwaka $\underline{a}^{\prime} w a k w$, Beau Dick la transforme en " happening politique " inscrit dans le présent où les protagonistes sont les Autochtones de la Colombie britannique et le gouvernement fédéral. On a là un exemple frappant de la manière dont des pratiques culturelles dites traditionnelles sont opérantes aujourd'hui pour stigmatiser les politiques néo-colonialistes du gouvernement canadien et son mépris des questions environnementales au profit de grandes compagnies pétrolières, par exemple. Ce type de performance, à la fois cérémonielle et politique, menée par Beau Dick et Gujaaw 
est d'autant plus important que certains artistes sont aussi des militants défenseurs notamment des causes environnementales ; en raison de leur notoriété ils sont considérés comme les porte-parole de leur communauté.

- Jean-Philippe Uzel. Les potlatchs, loin d'être des institutions « archaïques », comme les qualifiait Mauss, font aujourd'hui partie du mouvement d'affirmation des Premières Nations de la Colombie britannique (au même titre que le mouvement des pow-wow en Ontario et au Québec). Or, le monde de l'art contemporain occidental semble ne pas vouloir voir cette « contemporanéité » du potlatch. Ce déni serait-il, selon vous, une forme de néo-primitivisme?

- Sonny Assu. Le monde de l'art occidental refuse de voir dans I'« autre » (qu'il soit Noir, Asiatique ou autochtone) autre chose qu'un primitif. II ne s'agit pas de néo-primitivisme ; les gardiens du temple continuent simplement à mettre l'« autre » dans une boîte à étiqueter, il s'agit d'un défaut de compréhension.

- Marie Mauzé. L'organisation des potlatchs a progressivement repris une quinzaine d'années après la levée de leur interdiction en 1951. Ils ont connu un essor nouveau à partir de la fin des années 1970 et le début des années 1980, au moment où l'on a procédé à la réactualisation des titres et des privilèges familiaux. Le monde de l'art contemporain s'intéresse moins aux sociétés autochtones en tant que telles, au sort que leur a réservé la colonisation européenne depuis plusieurs siècles et qui a mené au quasi anéantissement de leur culture et de leur langue, à la confiscation de leurs territoires ancestraux, ni, d'ailleurs, à leur devenir et aux formes de résilience que ces sociétés ont su opposer au pouvoir colonial, qu'à l'appropriation d'un concept devenu, au travers de ses avatars, un concept vide. Ce déni est certainement une forme de néo-primitivisme, mais surtout le résultat d'une grande ignorance des enjeux auxquels font face les sociétés autochtones aujourd'hui.

- Charlotte Townsend-Gault. Mais en quoi l'intelligence collective du monde de l'art occidental peut-elle servir de guide à quoi que ce soit ? (La question fait une nouvelle fois pencher la balance en faveur de l'anthropologie.) Au lieu de néo-primitivisme, je parlerais plutôt de «néo-exotisme », qui semble susciter un grand intérêt.

L'histoire trouble des relations entre Autochtones et non-Autochtones au Canada est de mieux en mieux connue. Si l'on doit accueillir les excuses officielles et les gestes de réconciliation avec une bonne dose de scepticisme, ces signes d'ouverture relative servent parfois les intérêts des Premières Nations, ce que le doute et le cynisme ne doivent pas faire oublier. Les peuples autochtones ont maintenant voix au chapitre, les dégâts causés par le système des écoles résidentielles (ou pensionnats autochtones) sont reconnus, et on cherche, partout au Canada, à résoudre des problèmes chroniques.

J'ai pu constater que quantité de non-Autochtones du Canada ou d'ailleurs reconnaissent la contemporanéité du potlatch et d'autres modes cérémoniels ; mais cela exige un réajustement constant étant donné les orientations prises par diverses formes de protectionnisme des cultures autochtones. Le séparatisme farouche semble parfois s'opposer à la contemporanéité. Le phénomène n'est pas nécessairement négatif. II s'agit peut-être d'une forme de riposte à la classification et à la reclassification auxquelles ont été soumises en permanence quantité d'aspects de la culture autochtone.

- Rémi Labrusse. Je ne parlerais pas de néo-primitivisme en ce qui concerne le domaine de la création artistique et littéraire. Le néo-primitivisme serait plutôt du côté de l'histoire de l'art 
lorsqu'elle cherche à rapprocher en vrai, de force, le côté " potlatch » de l'art contemporain et les potlatchs amérindiens. Or, comme je l'ai suggéré, la totale indifférence de Debord, par exemple, à l'égard du potlatch « originel » relève plutôt d'un anti-primitivisme militant. Et ce qui est frappant dans les usages de l'idée de potlatch au sein de certains mondes de l'art actuel, c'est la marque quasi hégémonique de la trilogie Mauss - Bataille - Debord. Tout se passe comme si le potlatch était né dans ces zones de discours-là, sans autre arrière-fond ; comme si le sol originaire de " notre " potlatch, c'était l'Essai sur le don, La Part maudite et les textes de l'Internationale lettriste (en effaçant un peu Huizinga au passage, du reste). À l'opposé de ces usages artistiques actuels, le néo-primitivisme stricto sensu supposerait déjà de prendre en compte les potlatchs des Premières Nations, qu'ils soient anciens ou récents, en s'arrogeant le droit de leur distribuer ou non de la valeur.

Cela dit, l'universalisation du concept de potlatch, continûment orchestrée en Occident depuis Mauss, n'empêche pas les évolutions, entre les années 1950 et le début du XXIe siècle, ni les confusions conceptuelles, au sein des réalisations artistiques : autour du motif du potlatch s'emmêlent des horizons de fête communautaire, de critique institutionnelle et d'éthique post-chrétienne du don sans contrepartie. Sans parler du mot d'ordre médiatique de "l'esthétique relationnelle ", en faveur au début des années 2000, un artiste comme Thomas Hirschhorn, avec son idée d'un "public non exclusif ", évoque volontiers sa sympathie pour l'idée de potlatch et la relie automatiquement à Bataille, auquel il a consacré un de ses "Monuments", à Cassel, en 2002. En outre, sa démarche consiste à créer des situations éphémères de court-circuit social qui s'inspirent lointainement des discours situationnistes. D'un côté, la dimension agonistique perdure dans le défi que ses gestes artistiques adressent aux capacités intégratrices du capitalisme en général et du marché de l'art en particulier, confronté à des prestations - plutôt qu'à des productions - précaires et gratuites. D'un autre côté, à l'inverse de ce qui se passait chez Bataille ou Debord, le don y prend nettement le pas sur la destruction et cette morale du don - en dépit de la rhétorique de la violence à laquelle recourt volontiers l'artiste dans ses déclarations - s'enracine dans un socle compassionnel judéo-chrétien. Dans le même sillage, la valeur éthique centrale de ses projets est l'universalisme, qu'il oppose au communautarisme et à ce qu'il appelle l'obscurantisme.

- Jean-Philippe Uzel. Le témoignage visuel le plus complet du potlatch nous a été laissé par Edward S. Curtis dans son film de fiction In the Land of the Head Hunters (1914) qui a récemment fait l'objet d'une reconstruction / restauration en collaboration avec la nation Kwakwaka $\underline{\prime}^{\prime} w a k w$ (fig. 8). Pensez-vous que le "regard occidental ", avec toutes ses approximations, peut contribuer à la reconstruction et à l'affirmation culturelle des peuples autochtones?

- Charlotte Townsend-Gault. Ce « témoignage visuel » nous a été laissé par les Kwakwạkạ́wakw apparaissant dans le film, ou ayant collaboré avec Curtis à des titres divers. In the Land of the Head Hunters est un projet interculturel qui repose entièrement sur une collaboration ${ }^{26}$, et c'est aussi le cas des recherches ethnographiques de Mcllwraith sur les Bella Coola ou des travaux du linguiste Edward Sapir sur leur langue, travaux menés pour et avec les Nuu-chad-nulth ${ }^{27}$. La vision occidentale ne peut dominer qu'à condition d'escamoter la participation des peuples autochtones, ou d'oublier qu'ils avaient peut-être, tout comme leurs descendants, une conception différente des choses. Parmi les Kwakwaka $\underline{\prime}^{\prime} w a k w$, certains apprécient le travail de Curtis, d'autres le rejettent. On fait preuve d'une forme de mépris en croyant qu'ils seront tous du même avis. En outre, il n'y a plus grand intérêt désormais (si jamais ce fut le cas) à considérer que le « regard occidental » est fait d'un seul et même bloc. 


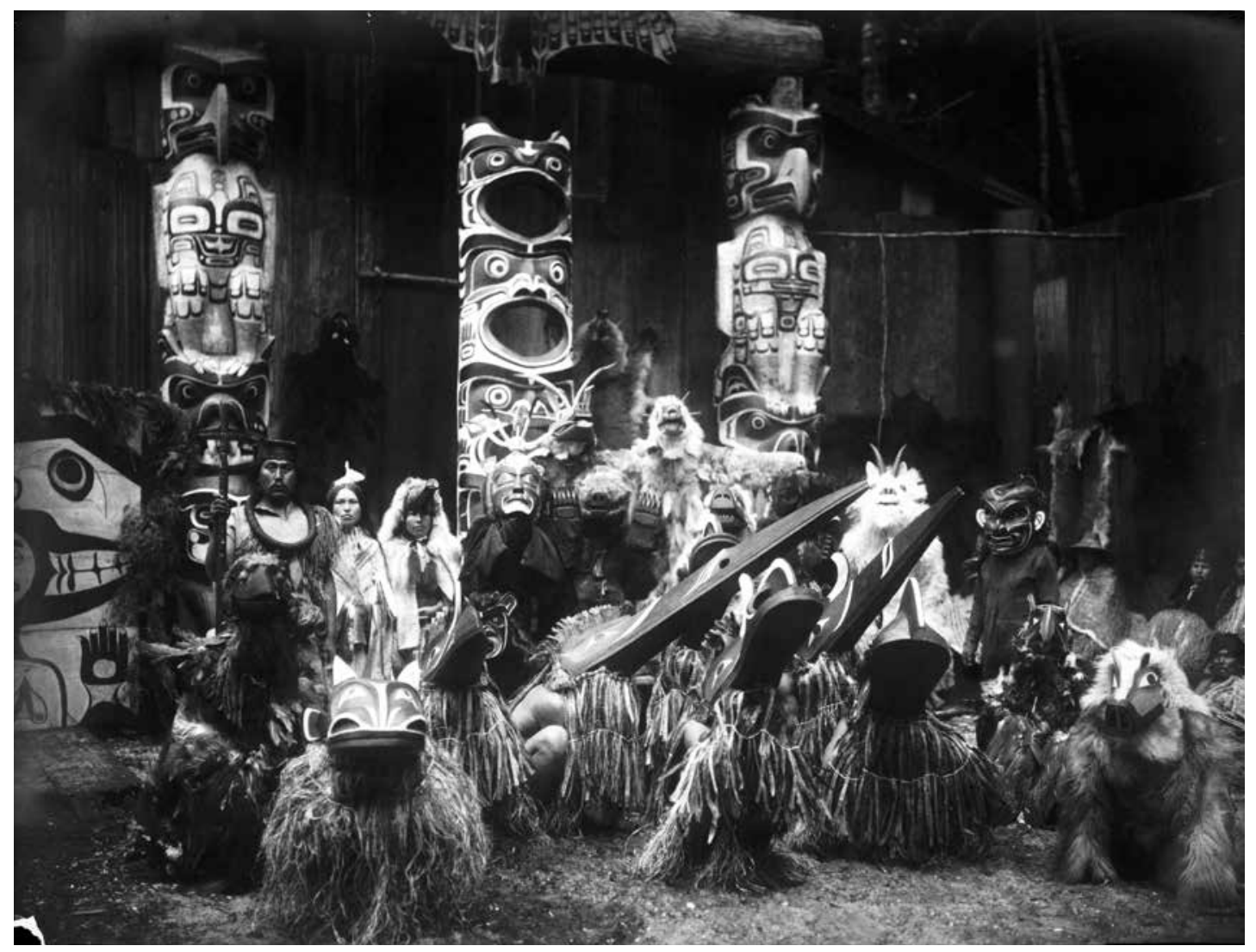

8. Edward S. Curtis, Masked dancers Qagyuhl, 1914, photogravure,

$31 \times 43 \mathrm{~cm}$, Washington, DC, Library of Congress, Prints and Photographs Division.

9a-b. Sonny Assu, 1884-1951 [a], et détail [b], 2009, 67 gobelets en cuivre repoussé au tour et couvercles avec couverture de Hudson's Bay (dimensions variables), collection Audain Art Museum.
Tous les " étrangers » ne sont pas des "Occidentaux ». La généralisation est souvent synonyme de banalisation, et il est douteux que l'on pourra remédier aux stéréotypes culturels en en invoquant d'autres.

Mes premières rencontres sur les territoires canadiens non encore colonisés, comme on les a appelés, ont été placés sous le signe de la coopération et de l'échange réciproque. Ils étaient marqués par un esprit d'ouverture, parfois sans contrainte protocolaire, et ce chez toutes les nations autochtones nord-américaines, dans toute leur diversité. Un esprit d'ouverture trahi par le régime colonial et ses notions bancales sur l'éducation et la

religion. Le juriste nuu-chah-nulth Douglas White et sa sœur n'ont tenu aucun compte des règlements des musées ni des questions de contemporanéité lorsqu'ils se sont mis à danser devant le rideau cérémoniel de leur famille exposé, dans toute sa splendeur, à l'American Museum of Natural History de New York. Dans "Where Mere Words Failed ${ }^{28}$ ", White raconte cette fusion des droits anciens et nouveaux des peuples autochtones qui a rendu contemporaine une ancienne cérémonie. Cela s'applique également à la pratique du potlatch. Le pow-wow contemporain, marqué par la générosité, I'humour et la gravité, est un bel exemple d'invitation qui s'adresse à tous, sans aucun sentimentalisme : prenez un morceau de bannock, dansez avec nous. Si l'on vous invite à un potlatch, acceptez de bon cœur. Tout le monde a beaucoup à apprendre, et c'est la raison d'être du potlatch, depuis toujours. 

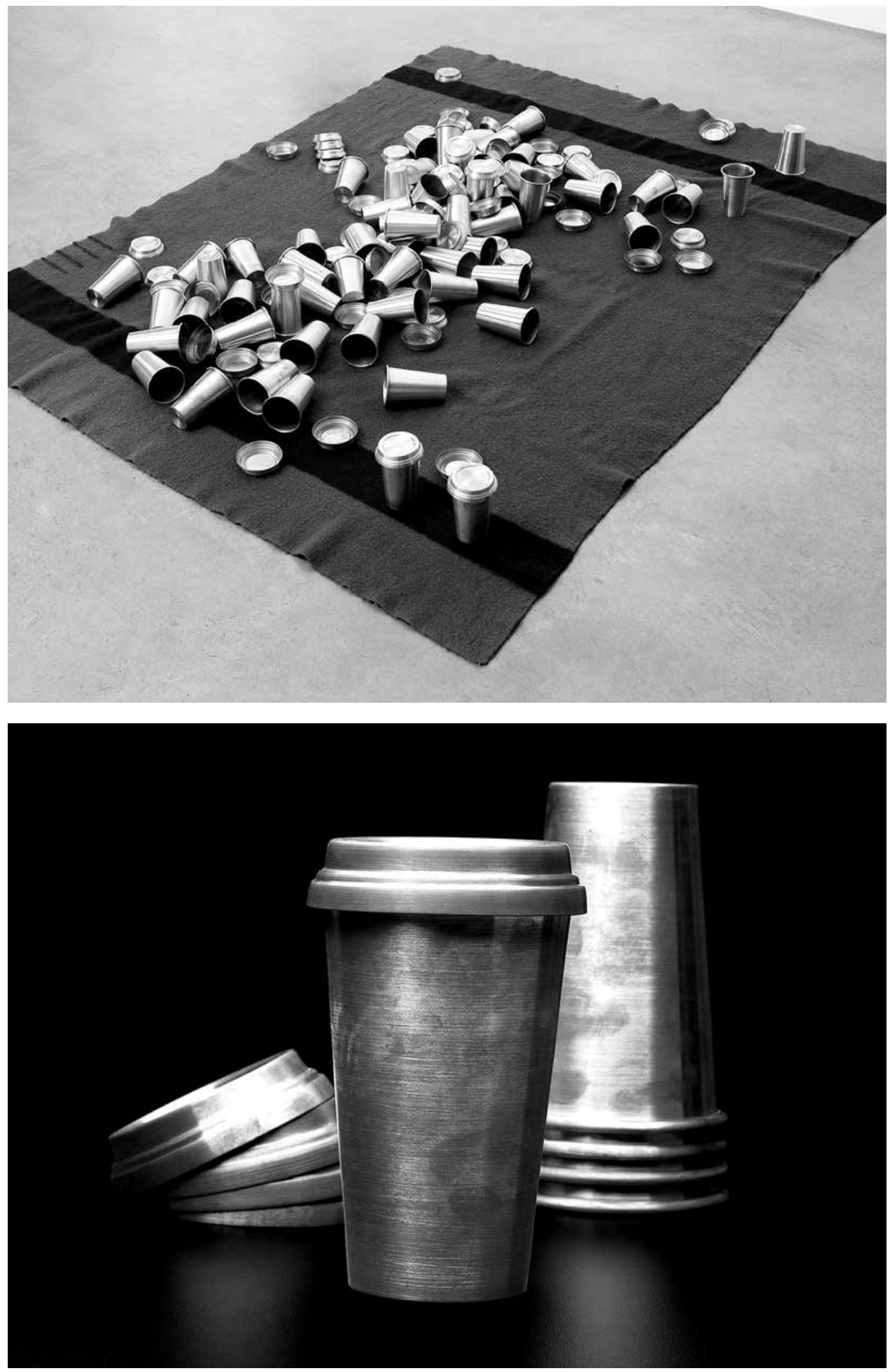
- Marie Mauzé. On ne peut pas dire que le film de Curtis, tourné en 1914, soit véritablement un témoignage de potlatch. C'est un film de fiction qui intègre certains aspects d'un potlatch organisé à l'occasion d'un mariage. À cette époque le potlatch était interdit. Les participants ont, en toute connaissance de cause, adhéré au projet, ce qui était considéré comme un acte de résistance, car ils voyaient là une occasion de donner à voir à un large public euro-canadien / américain certains aspects de leur riche héritage culturel avec la transmission de privilèges héréditaires, comme c'est le cas pour les danses cérémonielles. Bien que certains membres de la "nation » kwakwaka $\underline{a}^{\prime} w a k w$ aient été impliqués dans le processus de restauration du film de Curtis, le «produit » a fait l'objet de critiques de la part de représentants kwakwaka ${ }^{\prime}$ wakw qui n'ont pas autorisé la projection du film dans leur village. Quel que soit l'engagement des chercheurs non autochtones au bénéfice de la réappropriation de leur culture par les peuples autochtones, il n'en demeure pas moins que ces derniers cherchent, à raison, à rééquilibrer les relations de pouvoir entre eux et les « autres » et à contrôler leur image et les discours stéréotypés produits à leur endroit.

- Sonny Assu. Les peuples et les artistes autochtones n'ont pas besoin de la sanction d'un regard occidental / colonial pour produire des œuvres d'art, des textes ou de la théorie. II n'y a pas reconstitution, mais continuation (fig. 9a-b).

Les contributions de Sonny Assu

et Charlotte Townsend-Gault

ont été traduites par Françoise Jaouën. 


\section{Sonny Assu}

Sonny Assu mêle principes occidentaux et kwakwakka'wakw dans ses créations afin de raconter son histoire familiale et son vécu d'Autochtone dans I'État colonial du Canada. Ses œuvres figurent à la National Gallery du Canada, à l'Art Museum de Seattle, à la Vancouver Art Gallery, au Musée d'anthropologie de l'université de Colombie britannique, et dans diverses autres collections publiques et privées canadiennes, américaines ou britanniques.

\section{Charlotte Townsend-Gault}

Charlotte Townsend-Gault, professeure émérite du département d'histoire de l'art, d'art et de théorie visuelle de l'université de Colombie britannique est également professeure honoraire d'anthropologie à I'University College de Londres. Elle a publié de nombreux articles et ouvrages sur l'art des peuples autochtones canadiens et sa réception dans le contexte social contemporain. Elle a en outre dirigé diverses expositions : Born to Live and Die on Your Colonialist Reservations (consacrée à Lauwrence Paul Yuxwelptun), The Named and the Un-named (consacrée à Rebeccas Belmore) et Backstory, première exposition publique de thliitsapilthim (rideaux cérémoniels nuu-chah-nulth) qui s'est tenue à la Belkin Art Gallery de I'Université de Colombie britannique. Elle a également co-dirigé, avec Jennifer Kramer et Ki-ke-in, l'ouvrage Northwest Coast Native Art: An Anthology of Changing Ideas, récompensé en 2015 par le Canada Prize in the Humanities.

\section{Rémi Labrusse}

Rémi Labrusse enseigne I'histoire de l'art à l'université Paris Nanterre. Il a consacré une part importante de ses recherches à la valeur instrumentale, pour la construction de l'identité occidentale moderne, des notions d'Orient et de primitivité, de la fin du XIX ${ }^{\mathrm{e}}$ siècle à nos jours.

\section{Marie Mauzé}

Marie Mauzé est ethnologue, directrice de recherche au CNRS et membre du Laboratoire d'anthropologie sociale. Elle est spécialiste des Premières Nations de la côte nord-ouest et notamment des Kwakwaka' $\underline{a}^{\prime} w a k w$. Elle est l'auteure de plusieurs articles sur le regard des surréalistes porté sur l'art de la côte nord-ouest et de l'art yup'ik d'Alaska.

\section{Jean-Philippe Uzel}

Jean-Philippe Uzel est professeur d'histoire de l'art à l'université du Québec à Montréal et membre du CIÉRA - Centre interuniversitaire d'études et de recherches autochtones. Son champ d'expertise porte sur l'histoire et la théorie de l'art moderne et contemporain, et plus particulièrement sur les rapports entre art et politique. C'est sous cet angle qu'il s'intéresse depuis près d'une vingtaine d'années à l'art contemporain autochtone d'Amérique du Nord.

\section{NOTES}

1. Georges Bataille, "La notion de dépense », dans $L a$ critique sociale, $\mathrm{n}^{\circ}$ 7, 1933, p. 7-15; Georges Bataille, La part maudite: essai d'économie générale, Paris, Éditions de Minuit, 1949.

2. Harold Bloom, The Anxiety of Influence: A Theory of Poetry, New York, Oxford University Press, 1997.

3. Gilles Deleuze et Félix Guattari, Mille plateaux, paris, Éditions de Minuit, 1980, p. 10.

4. Claus Deimel, Sarah Elizabeth Holland, Jutta Charlotte von Bloch (dir.), The Power of Giving: Gifts in the Kwakwa ${ }^{2}$ a'wakw Big House from the Canadian Northwest Coast and at the Saxon rulers' court in Dresden, cat. exp. (Alert Bay, U'mista Cultural Centre / Dresde, Lipsiusbau, Staatliche Kunstsammlungen Dresden, 2011), Berlin, Deutscher Kunstverlag, 2011.

5. Gloria Cranmer Webster, "The Dark Years ", dans Charlotte Townsend-Gault, Jennifer Kramer, and Ki-kein (dir.), Native Art of the Northwest Coast: A History of Changing Ideas, Vancouver, University of British Columbia Press, 2013, p. 265-270.

6. Marcel Mauss, «Essai sur le don. Forme et raison de l'échange dans les sociétés archaïques", dans L'année sociologique, nouvelle série, $n^{\circ} 1,1925$, p. 30-186, réed. dans Sociologie et anthropologie, Paris, Presses universitaires de France (Sociologie d'aujourd'hui), 1950, p. $145-279$.

7. Bataille, 1933, cité n. 1 et Bataille, 1949, cité n. 1.

8. Claude Lévi-Strauss et Didier Eribon, De Près et de loin, Paris, Éditions Odile Jacob, p. 52.

9. Potlatch. Bulletin d'information de l'Internationale lettriste, nº 15, 22 décembre 1954.

10. Potlatch. Bulletin d'information de l'Internationale lettriste, $\mathrm{n}^{\circ}$ 1, 22 juin 1954.

11. "Pourquoi le lettrisme?", dans Potlatch. Bulletin $d^{\prime}$ information de l'Internationale lettriste, $\mathrm{n}^{\circ} 22,9$ septembre 1955.

12. Voir Marie Mauzé, "Boas, les Kwagul et le potlatch. Éléments pour une réévaluation ", dans L'Homme, $\mathrm{n}^{\circ} 100,1986$, p. 21-63.

13. Mauss, (1925) 1950, cité n. 6, p. 153.

14. Marcel Mauss, "Nord-ouest américain. L'honneur et le crédit », dans Mauss, (1925) 1950, cité n. 6, p. 200.

15. Marcel Schwob, Le Livre de Monelle, Paris, L. Chailley, 1894, cité par André Breton, "Alfred Jarry » (1918), Les Pas perdus, Paris, Gallimard, 1924, p. 62 (CEuvres complètes, Marguerite Bonnet, Philippe Bernier, Etienne-Alain Hubert, José Pierre [éd.], Paris, Gallimard [Bibliothèque de la pléiade], 1988, I, p. 224). 
16. Joan Miró cité par Adolphe Basler, Les Marges, 15 février 1927, repris dans Victoria Combalía, EI Descubrimiento de Miró. Miró y sus críticos 1918-1929, Barcelone, Ediciones Destino, 1990, p. 181.

17. Joan Miró cité par Maurice Raynal, Anthologie de la peinture en France de 1906 à nos jours, Paris, Montaigne, 1927, p. 34

18. Johan Huizinga, Homo ludens. Essai sur la fonction sociale du jeu, Cécile Seresia (trad. fra.), Paris, Gallimard (Tel), 1988 [éd. orig : Homo ludens, Haarlem, Tjeenk Willink, 1938].

19. William Beynon, Potlatch at Gitsegukla: William Beynon's 1945 Field Notebook, Marjorie M. Halpin et Margaret Seguin Anderson (éd.), Vancouver, UBC Press, 2000.

20. Leslie A. Robertson et le clan Kwagu'É Gixsam, Standing up with Ga'axsta'las: Jane Constance Cook and the Politics of Memory, Church and Custom, Vancouver, UBC Press, 2012.

21. Cécile R. Ganteaume, Officially Indian: Symbols That Define the United States, Colin G. Calloway (foreword), Paul Chaat Smith (afterword), publié à l'occasion de l'exposition Americans (Washington, DC, National Museum of the American Indian, 2017-2022), Washington, DC, National Museum of the American Indian, Smithsonian Institution / University of Minnesota Press, 2017.

22. Georges Duthuit, "Le don indien », dans Labyrinthe, $\mathrm{n}^{\circ} 18,1^{\text {er }}$ avril 1946.

23. Georges Duthuit, « Le don indien sur la côte nordouest de l'Amérique (Colombie britannique) » dans Représentation et présence. Premiers écrits et travaux 1923-1952, Paris, Flammarion, 1974, p. 315-319, ici p. 317.

24. Duthuit, 1974, cité n. 23, p. 319.

25. George Clutesi, Potlatch, Sidney (Columbie britannique), Gray's Publishing, 1969.

26. Ce point est très bien illustré dans Brad Evans et Aaron Glass (dir.), Return to the Land of the Head Hunters: Edward S. Curtis and the Making of Modern Cinema, Seattle, University of Washington Press, 2013.

27. Thomas F. Mcllwraith, The Bella Coola Indians, Toronto, University of Toronto Press, 1948, 2 vol.

28. Douglas S. White, "'Where Mere Words Failed': Northwest Coast Art and Law », dans Townsend-Gault, Kramer and Ki-ke-in, 2013, cité n. 5, p. 633-675. 